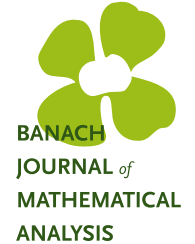

Banach J. Math. Anal. 10 (2016), no. 4, 800-814

http://dx.doi.org/10.1215/17358787-3649524

ISSN: $1735-8787$ (electronic)

http://projecteuclid.org/bjma

ANALYSIS

\title{
INEQUALITIES ON THE SPECTRAL RADIUS AND THE OPERATOR NORM OF HADAMARD PRODUCTS OF POSITIVE OPERATORS ON SEQUENCE SPACES
}

\author{
ROMAN DRNOVŠEK ${ }^{1}$ and ALJOŠA PEPERKO ${ }^{2 *}$
}

Communicated by F. Zhang

\begin{abstract}
K. M. R. Audenaert (2010), R. A. Horn and F. Zhang (2010), Z. Huang (2011), A. R. Schep (2011), A. Peperko (2012), and D. Chen and Y. Zhang (2015) have proved inequalities on the spectral radius and the operator norm of Hadamard products and ordinary matrix products of finite and infinite nonnegative matrices that define operators on sequence spaces. In the present article, we extend and refine several of these results, and we also prove some analogues for the numerical radius.
\end{abstract}

\section{INTRODUCTION}

In [20], X. Zhan conjectured that, for nonnegative $(n \times n)$-matrices $A$ and $B$, the spectral radius $\rho(A \circ B)$ of the Hadamard product satisfies

$$
\rho(A \circ B) \leq \rho(A B)
$$

where $A B$ denotes the usual matrix product of $A$ and $B$. This conjecture was confirmed by K. M. R. Audenaert in [1] by proving

$$
\rho(A \circ B) \leq \rho^{\frac{1}{2}}((A \circ A)(B \circ B)) \leq \rho(A B) .
$$

Copyright 2016 by the Tusi Mathematical Research Group.

Received Sep. 15, 2015; Accepted Jan. 25, 2016.

${ }^{*}$ Corresponding author.

2010 Mathematics Subject Classification. Primary 47B65; Secondary 15A42, 15 A60.

Keywords. Hadamard-Schur product, spectral radius, nonnegative matrices, positive operators, sequence spaces. 


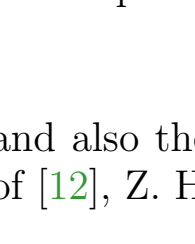

Banach J. Math. Anal. 10 (2016), no. 4, 800-814

http://dx.doi.org/10.1215/17358787-3649524

ISSN: $1735-8787$ (electronic)

http://projecteuclid.org/bjma

ANALYSIS

\title{
INEQUALITIES ON THE SPECTRAL RADIUS AND THE OPERATOR NORM OF HADAMARD PRODUCTS OF POSITIVE OPERATORS ON SEQUENCE SPACES
}

\author{
ROMAN DRNOVŠEK ${ }^{1}$ and ALJOŠA PEPERKO ${ }^{2 *}$
}

Communicated by F. Zhang

\begin{abstract}
K. M. R. Audenaert (2010), R. A. Horn and F. Zhang (2010), Z. Huang (2011), A. R. Schep (2011), A. Peperko (2012), and D. Chen and Y. Zhang (2015) have proved inequalities on the spectral radius and the operator norm of Hadamard products and ordinary matrix products of finite and infinite nonnegative matrices that define operators on sequence spaces. In the present article, we extend and refine several of these results, and we also prove some analogues for the numerical radius.
\end{abstract}

\section{INTRODUCTION}

In [20], X. Zhan conjectured that, for nonnegative $(n \times n)$-matrices $A$ and $B$, the spectral radius $\rho(A \circ B)$ of the Hadamard product satisfies

$$
\rho(A \circ B) \leq \rho(A B)
$$

where $A B$ denotes the usual matrix product of $A$ and $B$. This conjecture was confirmed by K. M. R. Audenaert in [1] by proving

$$
\rho(A \circ B) \leq \rho^{\frac{1}{2}}((A \circ A)(B \circ B)) \leq \rho(A B) .
$$

Copyright 2016 by the Tusi Mathematical Research Group.

Received Sep. 15, 2015; Accepted Jan. 25, 2016.

${ }^{*}$ Corresponding author.

2010 Mathematics Subject Classification. Primary 47B65; Secondary 15A42, 15 A60.

Keywords. Hadamard-Schur product, spectral radius, nonnegative matrices, positive operators, sequence spaces. 


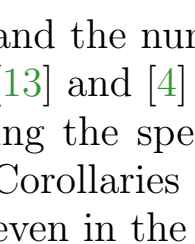

Banach J. Math. Anal. 10 (2016), no. 4, 800-814

http://dx.doi.org/10.1215/17358787-3649524

ISSN: $1735-8787$ (electronic)

http://projecteuclid.org/bjma

ANALYSIS

\title{
INEQUALITIES ON THE SPECTRAL RADIUS AND THE OPERATOR NORM OF HADAMARD PRODUCTS OF POSITIVE OPERATORS ON SEQUENCE SPACES
}

\author{
ROMAN DRNOVŠEK ${ }^{1}$ and ALJOŠA PEPERKO ${ }^{2 *}$
}

Communicated by F. Zhang

\begin{abstract}
K. M. R. Audenaert (2010), R. A. Horn and F. Zhang (2010), Z. Huang (2011), A. R. Schep (2011), A. Peperko (2012), and D. Chen and Y. Zhang (2015) have proved inequalities on the spectral radius and the operator norm of Hadamard products and ordinary matrix products of finite and infinite nonnegative matrices that define operators on sequence spaces. In the present article, we extend and refine several of these results, and we also prove some analogues for the numerical radius.
\end{abstract}

\section{INTRODUCTION}

In [20], X. Zhan conjectured that, for nonnegative $(n \times n)$-matrices $A$ and $B$, the spectral radius $\rho(A \circ B)$ of the Hadamard product satisfies

$$
\rho(A \circ B) \leq \rho(A B)
$$

where $A B$ denotes the usual matrix product of $A$ and $B$. This conjecture was confirmed by K. M. R. Audenaert in [1] by proving

$$
\rho(A \circ B) \leq \rho^{\frac{1}{2}}((A \circ A)(B \circ B)) \leq \rho(A B) .
$$

Copyright 2016 by the Tusi Mathematical Research Group.

Received Sep. 15, 2015; Accepted Jan. 25, 2016.

${ }^{*}$ Corresponding author.

2010 Mathematics Subject Classification. Primary 47B65; Secondary 15A42, 15 A60.

Keywords. Hadamard-Schur product, spectral radius, nonnegative matrices, positive operators, sequence spaces. 


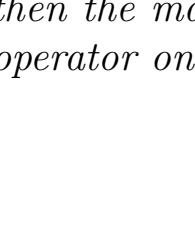

Banach J. Math. Anal. 10 (2016), no. 4, 800-814

http://dx.doi.org/10.1215/17358787-3649524

ISSN: $1735-8787$ (electronic)

http://projecteuclid.org/bjma

ANALYSIS

\title{
INEQUALITIES ON THE SPECTRAL RADIUS AND THE OPERATOR NORM OF HADAMARD PRODUCTS OF POSITIVE OPERATORS ON SEQUENCE SPACES
}

\author{
ROMAN DRNOVŠEK ${ }^{1}$ and ALJOŠA PEPERKO ${ }^{2 *}$
}

Communicated by F. Zhang

\begin{abstract}
K. M. R. Audenaert (2010), R. A. Horn and F. Zhang (2010), Z. Huang (2011), A. R. Schep (2011), A. Peperko (2012), and D. Chen and Y. Zhang (2015) have proved inequalities on the spectral radius and the operator norm of Hadamard products and ordinary matrix products of finite and infinite nonnegative matrices that define operators on sequence spaces. In the present article, we extend and refine several of these results, and we also prove some analogues for the numerical radius.
\end{abstract}

\section{INTRODUCTION}

In [20], X. Zhan conjectured that, for nonnegative $(n \times n)$-matrices $A$ and $B$, the spectral radius $\rho(A \circ B)$ of the Hadamard product satisfies

$$
\rho(A \circ B) \leq \rho(A B)
$$

where $A B$ denotes the usual matrix product of $A$ and $B$. This conjecture was confirmed by K. M. R. Audenaert in [1] by proving

$$
\rho(A \circ B) \leq \rho^{\frac{1}{2}}((A \circ A)(B \circ B)) \leq \rho(A B) .
$$

Copyright 2016 by the Tusi Mathematical Research Group.

Received Sep. 15, 2015; Accepted Jan. 25, 2016.

${ }^{*}$ Corresponding author.

2010 Mathematics Subject Classification. Primary 47B65; Secondary 15A42, 15 A60.

Keywords. Hadamard-Schur product, spectral radius, nonnegative matrices, positive operators, sequence spaces. 


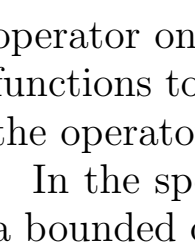

Banach J. Math. Anal. 10 (2016), no. 4, 800-814

http://dx.doi.org/10.1215/17358787-3649524

ISSN: $1735-8787$ (electronic)

http://projecteuclid.org/bjma

ANALYSIS

\title{
INEQUALITIES ON THE SPECTRAL RADIUS AND THE OPERATOR NORM OF HADAMARD PRODUCTS OF POSITIVE OPERATORS ON SEQUENCE SPACES
}

\author{
ROMAN DRNOVŠEK ${ }^{1}$ and ALJOŠA PEPERKO ${ }^{2 *}$
}

Communicated by F. Zhang

\begin{abstract}
K. M. R. Audenaert (2010), R. A. Horn and F. Zhang (2010), Z. Huang (2011), A. R. Schep (2011), A. Peperko (2012), and D. Chen and Y. Zhang (2015) have proved inequalities on the spectral radius and the operator norm of Hadamard products and ordinary matrix products of finite and infinite nonnegative matrices that define operators on sequence spaces. In the present article, we extend and refine several of these results, and we also prove some analogues for the numerical radius.
\end{abstract}

\section{INTRODUCTION}

In [20], X. Zhan conjectured that, for nonnegative $(n \times n)$-matrices $A$ and $B$, the spectral radius $\rho(A \circ B)$ of the Hadamard product satisfies

$$
\rho(A \circ B) \leq \rho(A B)
$$

where $A B$ denotes the usual matrix product of $A$ and $B$. This conjecture was confirmed by K. M. R. Audenaert in [1] by proving

$$
\rho(A \circ B) \leq \rho^{\frac{1}{2}}((A \circ A)(B \circ B)) \leq \rho(A B) .
$$

Copyright 2016 by the Tusi Mathematical Research Group.

Received Sep. 15, 2015; Accepted Jan. 25, 2016.

${ }^{*}$ Corresponding author.

2010 Mathematics Subject Classification. Primary 47B65; Secondary 15A42, 15 A60.

Keywords. Hadamard-Schur product, spectral radius, nonnegative matrices, positive operators, sequence spaces. 


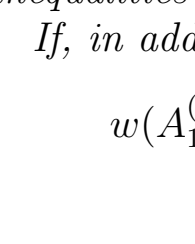

Banach J. Math. Anal. 10 (2016), no. 4, 800-814

http://dx.doi.org/10.1215/17358787-3649524

ISSN: $1735-8787$ (electronic)

http://projecteuclid.org/bjma

ANALYSIS

\title{
INEQUALITIES ON THE SPECTRAL RADIUS AND THE OPERATOR NORM OF HADAMARD PRODUCTS OF POSITIVE OPERATORS ON SEQUENCE SPACES
}

\author{
ROMAN DRNOVŠEK ${ }^{1}$ and ALJOŠA PEPERKO ${ }^{2 *}$
}

Communicated by F. Zhang

\begin{abstract}
K. M. R. Audenaert (2010), R. A. Horn and F. Zhang (2010), Z. Huang (2011), A. R. Schep (2011), A. Peperko (2012), and D. Chen and Y. Zhang (2015) have proved inequalities on the spectral radius and the operator norm of Hadamard products and ordinary matrix products of finite and infinite nonnegative matrices that define operators on sequence spaces. In the present article, we extend and refine several of these results, and we also prove some analogues for the numerical radius.
\end{abstract}

\section{INTRODUCTION}

In [20], X. Zhan conjectured that, for nonnegative $(n \times n)$-matrices $A$ and $B$, the spectral radius $\rho(A \circ B)$ of the Hadamard product satisfies

$$
\rho(A \circ B) \leq \rho(A B)
$$

where $A B$ denotes the usual matrix product of $A$ and $B$. This conjecture was confirmed by K. M. R. Audenaert in [1] by proving

$$
\rho(A \circ B) \leq \rho^{\frac{1}{2}}((A \circ A)(B \circ B)) \leq \rho(A B) .
$$

Copyright 2016 by the Tusi Mathematical Research Group.

Received Sep. 15, 2015; Accepted Jan. 25, 2016.

${ }^{*}$ Corresponding author.

2010 Mathematics Subject Classification. Primary 47B65; Secondary 15A42, 15 A60.

Keywords. Hadamard-Schur product, spectral radius, nonnegative matrices, positive operators, sequence spaces. 


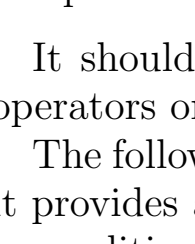

Banach J. Math. Anal. 10 (2016), no. 4, 800-814

http://dx.doi.org/10.1215/17358787-3649524

ISSN: $1735-8787$ (electronic)

http://projecteuclid.org/bjma

ANALYSIS

\title{
INEQUALITIES ON THE SPECTRAL RADIUS AND THE OPERATOR NORM OF HADAMARD PRODUCTS OF POSITIVE OPERATORS ON SEQUENCE SPACES
}

\author{
ROMAN DRNOVŠEK ${ }^{1}$ and ALJOŠA PEPERKO ${ }^{2 *}$
}

Communicated by F. Zhang

\begin{abstract}
K. M. R. Audenaert (2010), R. A. Horn and F. Zhang (2010), Z. Huang (2011), A. R. Schep (2011), A. Peperko (2012), and D. Chen and Y. Zhang (2015) have proved inequalities on the spectral radius and the operator norm of Hadamard products and ordinary matrix products of finite and infinite nonnegative matrices that define operators on sequence spaces. In the present article, we extend and refine several of these results, and we also prove some analogues for the numerical radius.
\end{abstract}

\section{INTRODUCTION}

In [20], X. Zhan conjectured that, for nonnegative $(n \times n)$-matrices $A$ and $B$, the spectral radius $\rho(A \circ B)$ of the Hadamard product satisfies

$$
\rho(A \circ B) \leq \rho(A B)
$$

where $A B$ denotes the usual matrix product of $A$ and $B$. This conjecture was confirmed by K. M. R. Audenaert in [1] by proving

$$
\rho(A \circ B) \leq \rho^{\frac{1}{2}}((A \circ A)(B \circ B)) \leq \rho(A B) .
$$

Copyright 2016 by the Tusi Mathematical Research Group.

Received Sep. 15, 2015; Accepted Jan. 25, 2016.

${ }^{*}$ Corresponding author.

2010 Mathematics Subject Classification. Primary 47B65; Secondary 15A42, 15 A60.

Keywords. Hadamard-Schur product, spectral radius, nonnegative matrices, positive operators, sequence spaces. 


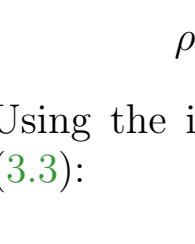

Banach J. Math. Anal. 10 (2016), no. 4, 800-814

http://dx.doi.org/10.1215/17358787-3649524

ISSN: $1735-8787$ (electronic)

http://projecteuclid.org/bjma

ANALYSIS

\title{
INEQUALITIES ON THE SPECTRAL RADIUS AND THE OPERATOR NORM OF HADAMARD PRODUCTS OF POSITIVE OPERATORS ON SEQUENCE SPACES
}

\author{
ROMAN DRNOVŠEK ${ }^{1}$ and ALJOŠA PEPERKO ${ }^{2 *}$
}

Communicated by F. Zhang

\begin{abstract}
K. M. R. Audenaert (2010), R. A. Horn and F. Zhang (2010), Z. Huang (2011), A. R. Schep (2011), A. Peperko (2012), and D. Chen and Y. Zhang (2015) have proved inequalities on the spectral radius and the operator norm of Hadamard products and ordinary matrix products of finite and infinite nonnegative matrices that define operators on sequence spaces. In the present article, we extend and refine several of these results, and we also prove some analogues for the numerical radius.
\end{abstract}

\section{INTRODUCTION}

In [20], X. Zhan conjectured that, for nonnegative $(n \times n)$-matrices $A$ and $B$, the spectral radius $\rho(A \circ B)$ of the Hadamard product satisfies

$$
\rho(A \circ B) \leq \rho(A B)
$$

where $A B$ denotes the usual matrix product of $A$ and $B$. This conjecture was confirmed by K. M. R. Audenaert in [1] by proving

$$
\rho(A \circ B) \leq \rho^{\frac{1}{2}}((A \circ A)(B \circ B)) \leq \rho(A B) .
$$

Copyright 2016 by the Tusi Mathematical Research Group.

Received Sep. 15, 2015; Accepted Jan. 25, 2016.

${ }^{*}$ Corresponding author.

2010 Mathematics Subject Classification. Primary 47B65; Secondary 15A42, 15 A60.

Keywords. Hadamard-Schur product, spectral radius, nonnegative matrices, positive operators, sequence spaces. 


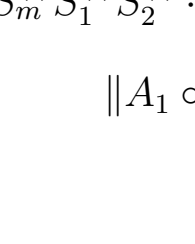

Banach J. Math. Anal. 10 (2016), no. 4, 800-814

http://dx.doi.org/10.1215/17358787-3649524

ISSN: $1735-8787$ (electronic)

http://projecteuclid.org/bjma

ANALYSIS

\title{
INEQUALITIES ON THE SPECTRAL RADIUS AND THE OPERATOR NORM OF HADAMARD PRODUCTS OF POSITIVE OPERATORS ON SEQUENCE SPACES
}

\author{
ROMAN DRNOVŠEK ${ }^{1}$ and ALJOŠA PEPERKO ${ }^{2 *}$
}

Communicated by F. Zhang

\begin{abstract}
K. M. R. Audenaert (2010), R. A. Horn and F. Zhang (2010), Z. Huang (2011), A. R. Schep (2011), A. Peperko (2012), and D. Chen and Y. Zhang (2015) have proved inequalities on the spectral radius and the operator norm of Hadamard products and ordinary matrix products of finite and infinite nonnegative matrices that define operators on sequence spaces. In the present article, we extend and refine several of these results, and we also prove some analogues for the numerical radius.
\end{abstract}

\section{INTRODUCTION}

In [20], X. Zhan conjectured that, for nonnegative $(n \times n)$-matrices $A$ and $B$, the spectral radius $\rho(A \circ B)$ of the Hadamard product satisfies

$$
\rho(A \circ B) \leq \rho(A B)
$$

where $A B$ denotes the usual matrix product of $A$ and $B$. This conjecture was confirmed by K. M. R. Audenaert in [1] by proving

$$
\rho(A \circ B) \leq \rho^{\frac{1}{2}}((A \circ A)(B \circ B)) \leq \rho(A B) .
$$

Copyright 2016 by the Tusi Mathematical Research Group.

Received Sep. 15, 2015; Accepted Jan. 25, 2016.

${ }^{*}$ Corresponding author.

2010 Mathematics Subject Classification. Primary 47B65; Secondary 15A42, 15 A60.

Keywords. Hadamard-Schur product, spectral radius, nonnegative matrices, positive operators, sequence spaces. 


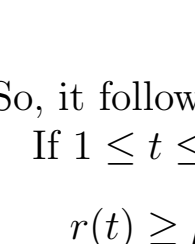

Banach J. Math. Anal. 10 (2016), no. 4, 800-814

http://dx.doi.org/10.1215/17358787-3649524

ISSN: $1735-8787$ (electronic)

http://projecteuclid.org/bjma

ANALYSIS

\title{
INEQUALITIES ON THE SPECTRAL RADIUS AND THE OPERATOR NORM OF HADAMARD PRODUCTS OF POSITIVE OPERATORS ON SEQUENCE SPACES
}

\author{
ROMAN DRNOVŠEK ${ }^{1}$ and ALJOŠA PEPERKO ${ }^{2 *}$
}

Communicated by F. Zhang

\begin{abstract}
K. M. R. Audenaert (2010), R. A. Horn and F. Zhang (2010), Z. Huang (2011), A. R. Schep (2011), A. Peperko (2012), and D. Chen and Y. Zhang (2015) have proved inequalities on the spectral radius and the operator norm of Hadamard products and ordinary matrix products of finite and infinite nonnegative matrices that define operators on sequence spaces. In the present article, we extend and refine several of these results, and we also prove some analogues for the numerical radius.
\end{abstract}

\section{INTRODUCTION}

In [20], X. Zhan conjectured that, for nonnegative $(n \times n)$-matrices $A$ and $B$, the spectral radius $\rho(A \circ B)$ of the Hadamard product satisfies

$$
\rho(A \circ B) \leq \rho(A B)
$$

where $A B$ denotes the usual matrix product of $A$ and $B$. This conjecture was confirmed by K. M. R. Audenaert in [1] by proving

$$
\rho(A \circ B) \leq \rho^{\frac{1}{2}}((A \circ A)(B \circ B)) \leq \rho(A B) .
$$

Copyright 2016 by the Tusi Mathematical Research Group.

Received Sep. 15, 2015; Accepted Jan. 25, 2016.

${ }^{*}$ Corresponding author.

2010 Mathematics Subject Classification. Primary 47B65; Secondary 15A42, 15 A60.

Keywords. Hadamard-Schur product, spectral radius, nonnegative matrices, positive operators, sequence spaces. 


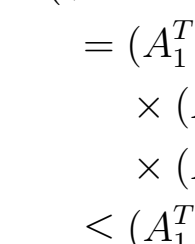

Banach J. Math. Anal. 10 (2016), no. 4, 800-814

http://dx.doi.org/10.1215/17358787-3649524

ISSN: $1735-8787$ (electronic)

http://projecteuclid.org/bjma

ANALYSIS

\title{
INEQUALITIES ON THE SPECTRAL RADIUS AND THE OPERATOR NORM OF HADAMARD PRODUCTS OF POSITIVE OPERATORS ON SEQUENCE SPACES
}

\author{
ROMAN DRNOVŠEK ${ }^{1}$ and ALJOŠA PEPERKO ${ }^{2 *}$
}

Communicated by F. Zhang

\begin{abstract}
K. M. R. Audenaert (2010), R. A. Horn and F. Zhang (2010), Z. Huang (2011), A. R. Schep (2011), A. Peperko (2012), and D. Chen and Y. Zhang (2015) have proved inequalities on the spectral radius and the operator norm of Hadamard products and ordinary matrix products of finite and infinite nonnegative matrices that define operators on sequence spaces. In the present article, we extend and refine several of these results, and we also prove some analogues for the numerical radius.
\end{abstract}

\section{INTRODUCTION}

In [20], X. Zhan conjectured that, for nonnegative $(n \times n)$-matrices $A$ and $B$, the spectral radius $\rho(A \circ B)$ of the Hadamard product satisfies

$$
\rho(A \circ B) \leq \rho(A B)
$$

where $A B$ denotes the usual matrix product of $A$ and $B$. This conjecture was confirmed by K. M. R. Audenaert in [1] by proving

$$
\rho(A \circ B) \leq \rho^{\frac{1}{2}}((A \circ A)(B \circ B)) \leq \rho(A B) .
$$

Copyright 2016 by the Tusi Mathematical Research Group.

Received Sep. 15, 2015; Accepted Jan. 25, 2016.

${ }^{*}$ Corresponding author.

2010 Mathematics Subject Classification. Primary 47B65; Secondary 15A42, 15 A60.

Keywords. Hadamard-Schur product, spectral radius, nonnegative matrices, positive operators, sequence spaces. 


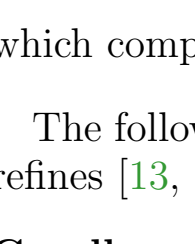

Banach J. Math. Anal. 10 (2016), no. 4, 800-814

http://dx.doi.org/10.1215/17358787-3649524

ISSN: $1735-8787$ (electronic)

http://projecteuclid.org/bjma

ANALYSIS

\title{
INEQUALITIES ON THE SPECTRAL RADIUS AND THE OPERATOR NORM OF HADAMARD PRODUCTS OF POSITIVE OPERATORS ON SEQUENCE SPACES
}

\author{
ROMAN DRNOVŠEK ${ }^{1}$ and ALJOŠA PEPERKO ${ }^{2 *}$
}

Communicated by F. Zhang

\begin{abstract}
K. M. R. Audenaert (2010), R. A. Horn and F. Zhang (2010), Z. Huang (2011), A. R. Schep (2011), A. Peperko (2012), and D. Chen and Y. Zhang (2015) have proved inequalities on the spectral radius and the operator norm of Hadamard products and ordinary matrix products of finite and infinite nonnegative matrices that define operators on sequence spaces. In the present article, we extend and refine several of these results, and we also prove some analogues for the numerical radius.
\end{abstract}

\section{INTRODUCTION}

In [20], X. Zhan conjectured that, for nonnegative $(n \times n)$-matrices $A$ and $B$, the spectral radius $\rho(A \circ B)$ of the Hadamard product satisfies

$$
\rho(A \circ B) \leq \rho(A B)
$$

where $A B$ denotes the usual matrix product of $A$ and $B$. This conjecture was confirmed by K. M. R. Audenaert in [1] by proving

$$
\rho(A \circ B) \leq \rho^{\frac{1}{2}}((A \circ A)(B \circ B)) \leq \rho(A B) .
$$

Copyright 2016 by the Tusi Mathematical Research Group.

Received Sep. 15, 2015; Accepted Jan. 25, 2016.

${ }^{*}$ Corresponding author.

2010 Mathematics Subject Classification. Primary 47B65; Secondary 15A42, 15 A60.

Keywords. Hadamard-Schur product, spectral radius, nonnegative matrices, positive operators, sequence spaces. 


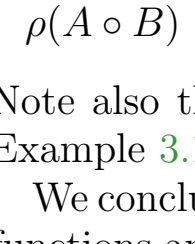

Banach J. Math. Anal. 10 (2016), no. 4, 800-814

http://dx.doi.org/10.1215/17358787-3649524

ISSN: $1735-8787$ (electronic)

http://projecteuclid.org/bjma

ANALYSIS

\title{
INEQUALITIES ON THE SPECTRAL RADIUS AND THE OPERATOR NORM OF HADAMARD PRODUCTS OF POSITIVE OPERATORS ON SEQUENCE SPACES
}

\author{
ROMAN DRNOVŠEK ${ }^{1}$ and ALJOŠA PEPERKO ${ }^{2 *}$
}

Communicated by F. Zhang

\begin{abstract}
K. M. R. Audenaert (2010), R. A. Horn and F. Zhang (2010), Z. Huang (2011), A. R. Schep (2011), A. Peperko (2012), and D. Chen and Y. Zhang (2015) have proved inequalities on the spectral radius and the operator norm of Hadamard products and ordinary matrix products of finite and infinite nonnegative matrices that define operators on sequence spaces. In the present article, we extend and refine several of these results, and we also prove some analogues for the numerical radius.
\end{abstract}

\section{INTRODUCTION}

In [20], X. Zhan conjectured that, for nonnegative $(n \times n)$-matrices $A$ and $B$, the spectral radius $\rho(A \circ B)$ of the Hadamard product satisfies

$$
\rho(A \circ B) \leq \rho(A B)
$$

where $A B$ denotes the usual matrix product of $A$ and $B$. This conjecture was confirmed by K. M. R. Audenaert in [1] by proving

$$
\rho(A \circ B) \leq \rho^{\frac{1}{2}}((A \circ A)(B \circ B)) \leq \rho(A B) .
$$

Copyright 2016 by the Tusi Mathematical Research Group.

Received Sep. 15, 2015; Accepted Jan. 25, 2016.

${ }^{*}$ Corresponding author.

2010 Mathematics Subject Classification. Primary 47B65; Secondary 15A42, 15 A60.

Keywords. Hadamard-Schur product, spectral radius, nonnegative matrices, positive operators, sequence spaces. 


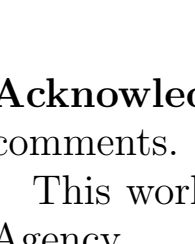

Banach J. Math. Anal. 10 (2016), no. 4, 800-814

http://dx.doi.org/10.1215/17358787-3649524

ISSN: $1735-8787$ (electronic)

http://projecteuclid.org/bjma

ANALYSIS

\title{
INEQUALITIES ON THE SPECTRAL RADIUS AND THE OPERATOR NORM OF HADAMARD PRODUCTS OF POSITIVE OPERATORS ON SEQUENCE SPACES
}

\author{
ROMAN DRNOVŠEK ${ }^{1}$ and ALJOŠA PEPERKO ${ }^{2 *}$
}

Communicated by F. Zhang

\begin{abstract}
K. M. R. Audenaert (2010), R. A. Horn and F. Zhang (2010), Z. Huang (2011), A. R. Schep (2011), A. Peperko (2012), and D. Chen and Y. Zhang (2015) have proved inequalities on the spectral radius and the operator norm of Hadamard products and ordinary matrix products of finite and infinite nonnegative matrices that define operators on sequence spaces. In the present article, we extend and refine several of these results, and we also prove some analogues for the numerical radius.
\end{abstract}

\section{INTRODUCTION}

In [20], X. Zhan conjectured that, for nonnegative $(n \times n)$-matrices $A$ and $B$, the spectral radius $\rho(A \circ B)$ of the Hadamard product satisfies

$$
\rho(A \circ B) \leq \rho(A B)
$$

where $A B$ denotes the usual matrix product of $A$ and $B$. This conjecture was confirmed by K. M. R. Audenaert in [1] by proving

$$
\rho(A \circ B) \leq \rho^{\frac{1}{2}}((A \circ A)(B \circ B)) \leq \rho(A B) .
$$

Copyright 2016 by the Tusi Mathematical Research Group.

Received Sep. 15, 2015; Accepted Jan. 25, 2016.

${ }^{*}$ Corresponding author.

2010 Mathematics Subject Classification. Primary 47B65; Secondary 15A42, 15 A60.

Keywords. Hadamard-Schur product, spectral radius, nonnegative matrices, positive operators, sequence spaces. 


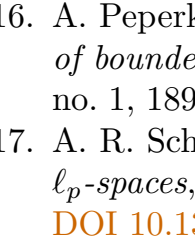

Banach J. Math. Anal. 10 (2016), no. 4, 800-814

http://dx.doi.org/10.1215/17358787-3649524

ISSN: $1735-8787$ (electronic)

http://projecteuclid.org/bjma

ANALYSIS

\title{
INEQUALITIES ON THE SPECTRAL RADIUS AND THE OPERATOR NORM OF HADAMARD PRODUCTS OF POSITIVE OPERATORS ON SEQUENCE SPACES
}

\author{
ROMAN DRNOVŠEK ${ }^{1}$ and ALJOŠA PEPERKO ${ }^{2 *}$
}

Communicated by F. Zhang

\begin{abstract}
K. M. R. Audenaert (2010), R. A. Horn and F. Zhang (2010), Z. Huang (2011), A. R. Schep (2011), A. Peperko (2012), and D. Chen and Y. Zhang (2015) have proved inequalities on the spectral radius and the operator norm of Hadamard products and ordinary matrix products of finite and infinite nonnegative matrices that define operators on sequence spaces. In the present article, we extend and refine several of these results, and we also prove some analogues for the numerical radius.
\end{abstract}

\section{INTRODUCTION}

In [20], X. Zhan conjectured that, for nonnegative $(n \times n)$-matrices $A$ and $B$, the spectral radius $\rho(A \circ B)$ of the Hadamard product satisfies

$$
\rho(A \circ B) \leq \rho(A B)
$$

where $A B$ denotes the usual matrix product of $A$ and $B$. This conjecture was confirmed by K. M. R. Audenaert in [1] by proving

$$
\rho(A \circ B) \leq \rho^{\frac{1}{2}}((A \circ A)(B \circ B)) \leq \rho(A B) .
$$

Copyright 2016 by the Tusi Mathematical Research Group.

Received Sep. 15, 2015; Accepted Jan. 25, 2016.

${ }^{*}$ Corresponding author.

2010 Mathematics Subject Classification. Primary 47B65; Secondary 15A42, 15 A60.

Keywords. Hadamard-Schur product, spectral radius, nonnegative matrices, positive operators, sequence spaces. 